\section{(6) OPEN ACCESS}

\title{
Atrial fibrillation associated with ivabradine treatment: meta-analysis of randomised controlled trials
}

\author{
Ruairidh I R Martin, ${ }^{1,2}$ Oksana Pogoryelova, ${ }^{1}$ Mauro Santibáñez Koref, ${ }^{1}$ \\ John P Bourke, ${ }^{2}$ M Dawn Teare, ${ }^{3}$ Bernard D Keavney ${ }^{1,4}$
}

- Additional material is published online only. To view please visit the journal online (http://dx.doi.org/10.1136/ heartjnl-2014-305482).

${ }^{1}$ Institute of Genetic Medicine, Newcastle University, Newcastle upon Tyne, UK ${ }^{2}$ Department of Cardiology, Freeman Hospital, Newcastle, UK

${ }^{3}$ School of Health and Related Research, University of Sheffield, Sheffield, UK ${ }^{4}$ Institute of Cardiovascular Sciences, University of Manchester, Manchester, UK

\section{Correspondence to} Dr Ruairidh Martin, Institute of Genetic Medicine, Newcastle University, Newcastle upon Tyne NE1 3BZ, UK; ruairidh. martin@newcastle.ac.uk

Received 8 January 2014 Revised 28 May 2014 Accepted 29 May 2014 Published Online First 20 June 2014

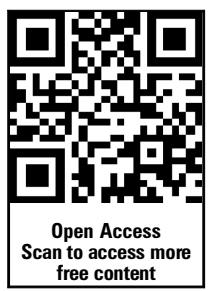

\section{SLinked}

- http://dx.doi.org/10.1136/ heartjnl-2014-306287

\section{CrossMark}

To cite: Martin RIR, Pogoryelova 0, Santibáñez Koref $\mathrm{M}$, et al. Heart 2014;100:1506-1510.

\section{ABSTRACT}

Objective To quantify any risk of atrial fibrillation (AF) associated with ivabradine treatment by meta-analysis of clinical trial data.

Methods Medline, Embase, Web of Knowledge and the Cochrane central register of controlled trials were searched for double-blinded randomised controlled trials of ivabradine with a minimum follow-up period of 4 weeks. For studies where AF data were unpublished, safety data were obtained from the European Medicines Agency (EMeA) website and personal communications. Studies were appraised for risk of bias using components recommended by the Cochrane Collaboration. Metaanalyses were performed of relative risk of $A F$ and absolute risk difference of $A F$ per year of treatment. The main outcome measure was incident AF during the follow-up period.

Results AF data were available from 11 studies: one from the published report, six from the EMeA and four from personal communications. Ivabradine treatment was associated with a relative risk of $A F$ of $1.15(95 \% \mathrm{Cl}$ 1.07 to $1.24, p=0.0027$ ) among 21571 patients in the meta-analysis. From this we estimated that the number needed to harm for ivabradine would be 208 (95\% Cl 122 to 667) per year of treatment.

Conclusions AF is a substantially more common side effect of ivabradine treatment than one patient in 10000 , the risk presently reported in the product literature. The incidence of AF has not routinely been reported in clinical trials of ivabradine.

\section{INTRODUCTION}

Ivabradine (Procoralan, Servier) is a heart rate lowering drug which acts by specifically inhibiting the pacemaker $\mathrm{I}_{f}$ current, which causes spontaneous depolarisation in the sino-atrial node that regulates the heart rate. ${ }^{1}$ Ivabradine was approved for use by the European Medicines Agency (EMeA) in 2005 for use in the treatment of stable angina pectoris in patients with normal sinus rhythm who are not able to tolerate $\beta$-blocker therapy. In 2010, the indication was extended to include treatment in patients with uncontrolled angina symptoms and a heart rate in excess of $60 \mathrm{bpm}$ despite $\beta$-blocker therapy, following the results of the BEAUTIFUL trial. ${ }^{2}$ A new indication was approved in 2012, following the results of the SHIFT trial, for treatment of chronic heart failure (New York Heart Association class II-IV) with systolic dysfunction, in patients in sinus rhythm and whose heart rate is greater than $75 \mathrm{bpm}$, in combination with standard therapy, including $\beta$-blocker therapy, or when $\beta$-blockers are contraindicated or not tolerated. ${ }^{3}$ Use of ivabradine is yet to be approved by the US Food and Drug Administration.

Genome-wide association studies have identified associations between genetic variants in the region of the gene HCN4, which codes for the main ion channel responsible for the $\mathrm{I}_{f}$ current, and both heart rate and atrial fibrillation (AF). ${ }^{4} 5$ The pulmonary venous myocardium, which is an important source of AF initiation and maintenance, ${ }^{6}$ demonstrates an $\mathrm{I}_{f}$ current which is affected by ivabradine. $^{7}$ This raises the possibility that ivabradine treatment may affect $\mathrm{AF}$ risk. $\mathrm{AF}$ is common in patients with coronary artery disease and cardiac failure $^{8}$ and so incident $\mathrm{AF}$ in the target population for ivabradine treatment would not necessarily be attributed to the drug. It has been shown that genome-wide association study hits can identify therapeutic targets and provide insight into drug side effects. For example, a single nucleotide plymorphism (SNP) in the tumour necrosis factor receptor 1 gene which is associated with multiple sclerosis (MS) risk has been shown to explain the mechanism whereby antitumour necrosis factor therapies typically exacerbate MS. ${ }^{9}$ We therefore examined whether there was a significant increase in AF risk associated with ivabradine treatment by meta-analysis, and quantified that risk in terms of number needed to harm $(\mathrm{NNH})$ per year.

\section{METHODS}

The study was performed in line with the recommendations of the Preferred Reporting Items for Systematic Reviews and Meta-Analyses (PRISMA) statement. $^{10}$

\section{Eligibility criteria, information sources and search protocol}

We included in our analysis all randomised controlled double-blind trials of ivabradine, for any indication and of any size, which followed patients up for at least 4 weeks. We did not exclude studies where AF incidence was not reported in the published manuscript, but attempted to identify that data wherever possible. We included both placebocontrolled studies and non-inferiority studies where an alternative drug treatment was used as a comparator. We excluded open or single-blinded studies, studies where the follow-up period was less 
than 4 weeks and studies where there was significant risk of bias, as assessed by the methods recommended by the Cochrane Collaboration.

We performed a systematic search, without language restriction, for randomised clinical trials of ivabradine treatment for any indication, using the search term 'ivabradine' and the document type 'clinical trial' or 'randomized controlled trial'. We searched Medline, Embase and Web of Knowledge from 1980 to October 2013, and the Cochrane Central Register of Controlled Trials. We also examined the reference lists of published trials, review articles and meta-analyses to identify other eligible trials. We read the scientific discussions of the EMeA to identify safety data that were not published in the original trial report. We also contacted directly the authors and in the case of industry-sponsored trials, the sponsors of studies which did not report the AF incidence in the original manuscript.

\section{Selection and quality assessment}

Studies were assessed on the basis of their title or abstract and those studies which appeared to meet the eligibility criteria were selected for full text review. Trials were assessed for eligibility and risk of bias using the components recommended by the Cochrane Collaboration: sequence generation, allocation concealment, blinding of participants, personnel and outcome assessors, incomplete outcome data, selective outcome reporting and other sources of bias. ${ }^{11}$ We considered any trial with a high or unclear risk of bias, by the Cochrane criteria, in sequence generation, allocation concealment and blinding to be at high risk of bias; all other studies were considered to be at low risk of bias.

\section{Outcome measures and data collected}

The main outcome measure was incident AF reported during the trial follow-up period. Where data on AF incidence were not reported, we contacted the authors by email on at least two occasions. We also identified AF incidence reported in the EMeA scientific discussions where trials were submitted as evidence for licensing and, in the case of trials sponsored by the manufacturer, contacted the manufacturer directly. Study data were collected on data collection forms which recorded reference data, ethical approval, randomisation, blinding, control agent (placebo or other drug), follow-up duration, inclusion and exclusion criteria and sponsorship and funding information as well as numbers of patients in the ivabradine and control arms with incident AF.

\section{Statistical analyses}

We performed meta-analysis of intention to treat outcomes using a random effect model in the metafor package in $\mathrm{R}$ statistical software V.3.0.2. ${ }^{12} 13$ Detailed statistical methods are available in the online supplement.

\section{RESULTS}

\section{Study selection and characteristics of included trials}

Our initial search strategy identified 84 published articles. We reviewed titles and abstracts and excluded papers that were not clinical trials, were not of sufficient duration of follow-up or which reported supplementary data from trials published elsewhere. We assessed the full text of the remaining 34 articles for eligibility. Eight papers were translated into English. After studies were excluded on the basis of study design (one), follow-up duration (two), randomisation (nine), study blinding (10) and duplication (two), our search strategy yielded 10 double-blind randomised controlled trials that enrolled 20022 patients for a mean of 1.5 (range 0.076-1.88) years follow-up, for a total of 30090 patient-years of follow-up (table 1, figure 1). ${ }^{2}{ }^{14-22}$ Risk of bias of the included studies is summarised in online supplementary table S1. Of the 10 studies identified, AF incidence was only reported in one, the SHIFT trial. We wrote to the authors of the other studies to request the unpublished AF data. Of the five studies not sponsored by the manufacturer, four authors replied with AF data. None of the authors of studies sponsored by the manufacturer provided AF data. We contacted the manufacturer directly who declined to provide the unpublished data, citing ongoing inhouse analyses. Of the missing studies, full AF safety data were provided for the BEAUTIFUL study on the EMeA website. The safety data from the ASSOCIATE study which is reported in the EMeA documentation do not report the incidence of AF. The data from the INITIATIVE

Table 1 Eligible trials for which AF data were sought

\begin{tabular}{|c|c|c|c|c|c|c|c|}
\hline Author & Year & Acronym & $\begin{array}{l}\text { Number of } \\
\text { participants }\end{array}$ & Indication & Control & $\begin{array}{l}\text { Ivabradine } \\
\text { dose (bid) }\end{array}$ & AF data source \\
\hline Tardif et $a l^{14}$ & 2005 & INITIATIVE & 939 & Angina & Atenolol & $7.5 \mathrm{mg} / 10 \mathrm{mg}$ & Included in 00SS \\
\hline Ruzyllo et al ${ }^{15}$ & 2007 & & 1195 & Angina & Amlodipine & $7.5 \mathrm{mg} / 10 \mathrm{mg}$ & Included in 00Ss \\
\hline Fox et $a l^{2}$ & 2008 & BEAUTIFUL & 10907 & Heart Failure & Placebo & $5 \mathrm{mg} / 7.5 \mathrm{mg}$ & EMeA \\
\hline Tardif et al ${ }^{16}$ & 2009 & ASSOCIATE & 889 & Angina & Placebo & $5 \mathrm{mg} / 7.5 \mathrm{mg}$ & No data \\
\hline Fasullo et al $l^{17}$ & 2009 & & 155 & Anterior STEMI & Metoprolol & $5 \mathrm{mg} / 7.5 \mathrm{mg}$ & No data \\
\hline Swedberg et $a l^{3}$ & 2010 & SHIFT & 6492 & Heart Failure & Placebo & $2.5-7.5 \mathrm{mg}$ & Original paper \\
\hline Nerla et al ${ }^{18}$ & 2012 & & 61 & Type II diabetes & Atenolol/placebo & $5 \mathrm{mg}$ & Personal communication \\
\hline $\begin{array}{l}\text { Dominguez- } \\
\text { Rodriguez et } \text { al }\end{array}$ & 2012 & & 27 & NSTE-ACS & Placebo & $5 \mathrm{mg}$ & Personal communication \\
\hline Cappato et $a l^{21}$ & 2012 & & 21 & IST & Placebo & $5 \mathrm{mg}$ & Personal communication \\
\hline Villano et $a l^{22}$ & 2013 & & 46 & Microvascular angina & Ranolazine/placebo & $5 \mathrm{mg}$ & Personal communication \\
\hline EMeA & 2005 & ooss & 3936 & & Atenolol/amlodipine/placebo & $5-10 \mathrm{mg}$ & EMeA \\
\hline
\end{tabular}

The AF data for the INITIATIVE study and from Ruzyllo et al were not available separately, but were included in the OOSS from the EMeA with three other studies.

AF, atrial fibrillation; bid, twice daily; EMeA, European Medicines Agency; IST, inappropriate sinus tachycardia; NSTE-ACS, non-ST elevation acute coronary syndrome; OOSS, overall oral safety set; STEMI, ST elevation myocardial infarction.

Trial acronyms: ASSOCIATE: Efficacy of the If current inhibitor ivabradine in patients with chronic stable angina receiving beta-blocker therapy: a 4 month, randomized, placebocontrolled trial. BEAUTIFUL:Ivabradine for patients with stable coronary artery disease and left ventricular dysfunction (BEAUTIFUL): a randomized, doube-blind, placebo-controlled trial. INITIATIVE: Efficacy of ivabradine, a new selective If inhibitor, compared with atenolol in patients with chronic stable angina. SHIFT: Systolic Heart Failure Treatments with If Inhibitor

Ivabradine Trial. 


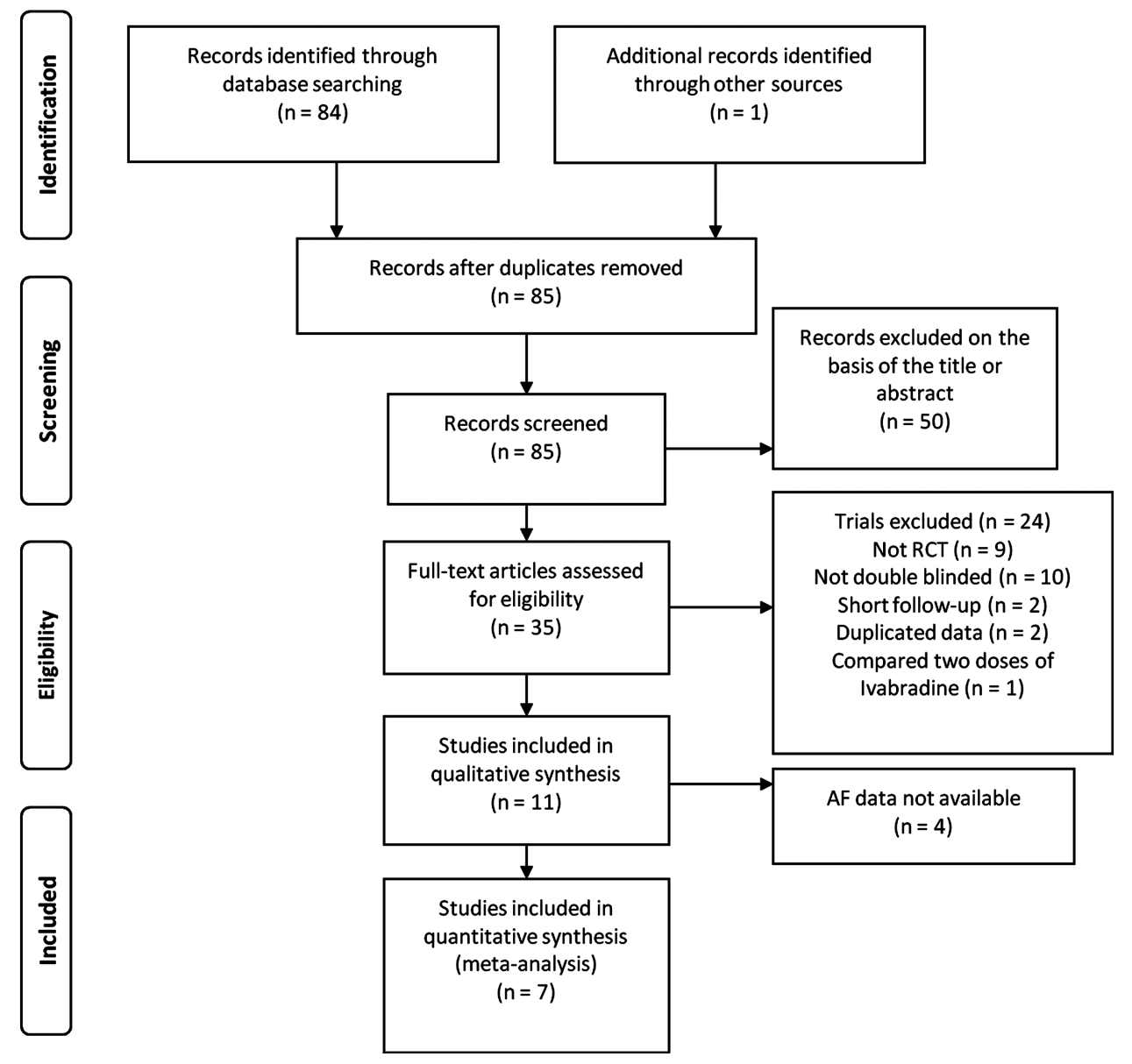

Figure 1 Flow chart of study selection.

study and from Ruzyllo et al are included in the analyses performed by the EMeA for licensing of ivabradine. The data are not available for each trial individually, however, but are combined with the safety data from a 3-month phase III clinical trial (for which we were unable to identify a published report) and two very short (four doses and 2 weeks, respectively) dose-ranging phase II studies. All of the studies included in this overall oral safety set (OOSS) were randomised, controlled and doubleblinded. None of the trials for which we obtained AF incidence data separately were included in the OOSS. In order to provide the most accurate estimate of the effect of $\mathrm{AF}$, we decided to include the OOSS as a single study in our meta-analysis and also to perform two sensitivity analyses. In the first, the OOSS was excluded and in the second only those trials where the drug treatment was compared with placebo, rather than an alternative such as amlodipine or a $\beta$-blocker, were included.

In our full data set for analysis including the OOSS, therefore, there were 21571 patients, followed up for a mean of 1.43 years to provide a total of 30755 patient-years of follow-up. When the OOSS was excluded, the data set included 17635 patients, followed up for a mean of 1.67 years to give a total of 29385 patient-years of follow-up. In the placebocontrolled trials only data set, there were 17571 patients followed up for a mean of 1.67 years to give a total of 29380 patient-years of follow-up.

\section{AF incidence}

Compared with controls, treatment with ivabradine was associated with a relative risk (RR) of $\mathrm{AF}$ of 1.15 times that of alternative treatments (95\% CI 1.07 to $1.24, \mathrm{p}=0.0027$ ) (figure 2). The results were largely similar if the OOSS was excluded, $\mathrm{RR}=1.15$ (95\% CI 1.06 to $1.25, \mathrm{p}=0.0065)$ and if only placebo-controlled trials were examined, $\mathrm{RR}=1.15$ (95\% CI 1.05 to $1.26, \mathrm{p}=0.015)$. When treatment indication was included in the model, the association between treatment indication and AF risk was not significant $(p=0.99)$, although this analysis was limited as the majority of patient-years of follow-up $(17475 / 17635)$ were in the two trials (SHIFT and BEAUTIFUL) for which the main treatment indication was heart failure.

When the absolute risk of AF was compared between ivabradine and control groups, ivabradine treatment was associated with an NNH of 208.3 per year of treatment (95\% CI 122.0 to 666.7, $\mathrm{p}=0.013$ ) (figure 3). This corresponds to an absolute risk difference of $0.48 \%$. This effect was very similar when the OOSS was excluded, $\mathrm{NNH}=208.3$ (95\% CI 113.6 to 1250 , $\mathrm{p}=0.028)$ and when only placebo-controlled trials were included, $\mathrm{NNH}=208.3$ (95\% CI 105.3 to $10000, \mathrm{p}=0.048$ ). As ivabradine is indicated for chronic treatment of angina or cardiac failure and treatment is likely to continue for more than 1 year, this $\mathrm{NNH}$ represents a substantially greater risk than the very rare (less than $1: 10000$ ) risk of $\mathrm{AF}$ reported in the product literature.

The heterogeneity of RR in the trials included was low, $\mathrm{I}^{2}=0.00 \%$, with the observed heterogeneity explained by within-study variance $(p=0.96)$. The same was true in the analysis of absolute risk difference, $\mathrm{I}^{2}=0.00 \%$, where the residual heterogeneity after accounting for length of follow-up was 


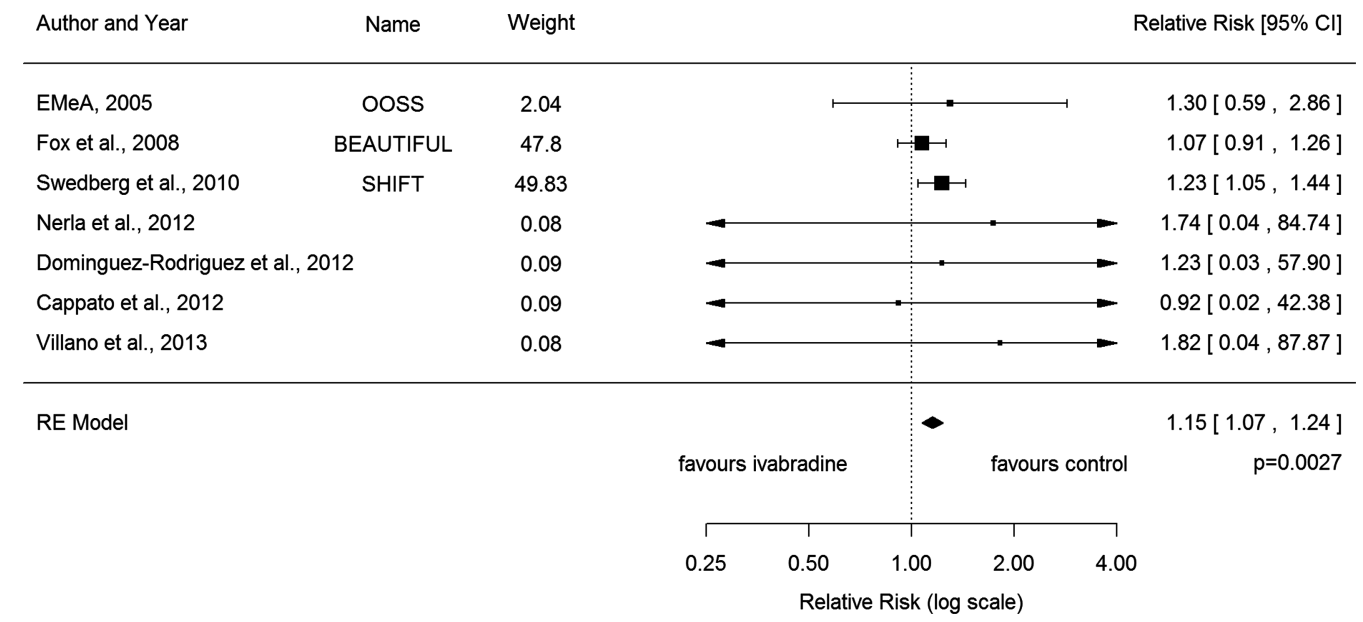

Figure 2 Forest plot of relative risk of atrial fibrillation in all trials with available data. RE Model, random effects model.

explained by within-study variance $(\mathrm{p}=0.86)$. This explains the similarity of the meta-analysis results between subgroups of trials.

There was no evidence of publication bias, rank test $p=1.00$ and regression test $p=0.64$, and examination of the funnel plots did not suggest any missing data (figure 4).

\section{DISCUSSION}

The results of this study, which used as much of the safety data as were available, quantify the risk of AF associated with ivabradine treatment. Use of ivabradine is associated with an approximately $15 \%$ increase in the risk of developing AF. This risk is greater than previously reported in the product safety information.

$\mathrm{AF}$ is a common complication of coronary artery disease and cardiac failure. Patients with these conditions have a higher rate of complications from $\mathrm{AF}$ than patients with $\mathrm{AF}$ alone. ${ }^{23}$ In addition, ivabradine has not been shown to limit the ventricular rate in patients in $\mathrm{AF}$, and so those patients who develop $\mathrm{AF}$ are likely to have higher heart rates, thus negating any benefits of ivabradine as a rate-lowering therapy in treating either angina or cardiac failure. These risks however need to be balanced against the benefits of ivabradine. In the SHIFT study, ivabradine

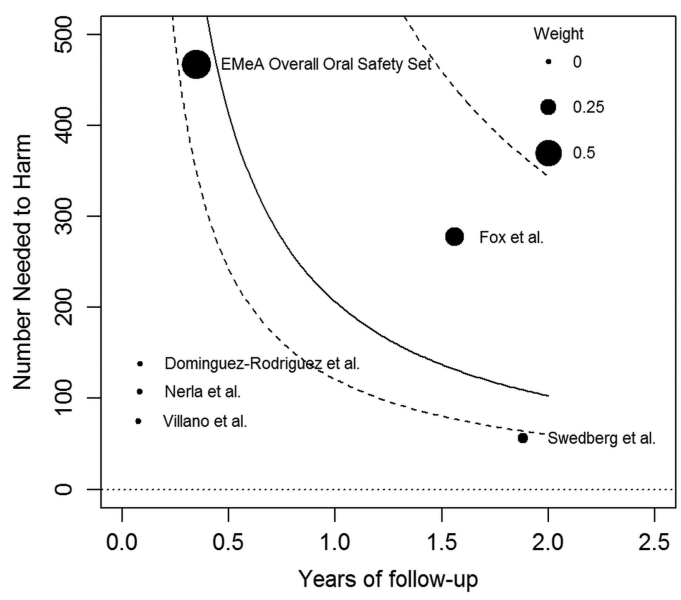

Figure 3 Number needed to harm per year of follow-up. Individual studies are plotted as circles with size proportional to the weighting in the meta-analysis model of absolute risk difference. The best fit line with $95 \% \mathrm{Cl}$ (dashed lines) is shown. treatment resulted in a reduction in the composite endpoint of death or hospitalisation with worsening heart failure; the number needed to treat (NNT) was 26, which was largely driven by a reduction in hospitalisations. ${ }^{3}$ A combined analysis of the individual data from the SHIFT trial and those patients in the BEAUTIFUL trial with a baseline heart rate $\geq 70 \mathrm{bpm}$ also identified a reduction in the same endpoint; the NNT was 40.7. ${ }^{24}$ Interestingly, in the same analysis, which looked at patients with a higher baseline heart rate, the increase in $\mathrm{AF}$ incidence was greater than we found in our meta-analysis; the NNH was 58 (albeit over a longer follow-up of 19-22 months). This raises the possibility that it is the patient group with the most to gain from ivabradine treatment who have the greatest risk of developing $\mathrm{AF}$ and that the absolute increase in the risk of AF with ivabradine treatment in this group is of a similar size to the absolute decrease in risk of hospitalisation.

The associations between genetic markers near HCN4, combined with the results of this meta-analysis, which identifies an association between $\mathrm{I}_{f}$ current inhibition and AF, provide further evidence for the role of HCN4 and the $\mathrm{I}_{f}$ current in the pathophysiology of AF. The results of this analysis also highlight the importance of large safety data sets and ongoing postmarketing surveillance of licensed and approved medications to identify side

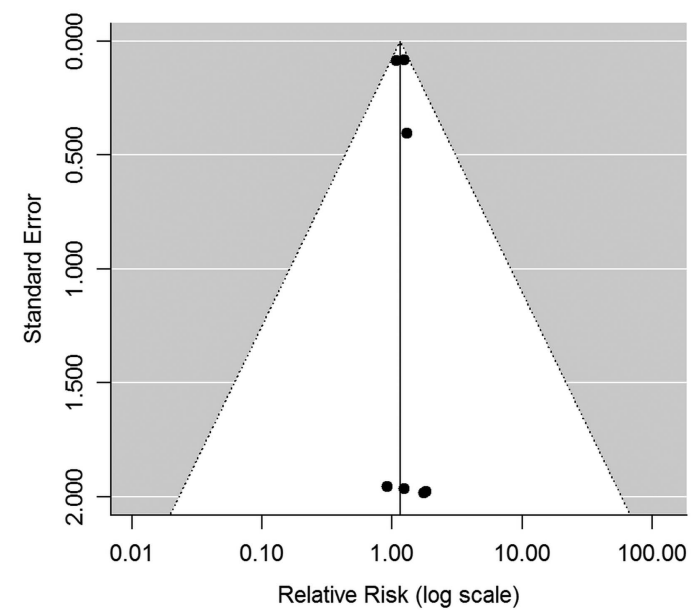

Figure 4 Funnel plot of relative risk versus SE. The plot is largely symmetrical, suggesting that there is no publication bias. 
effects which might otherwise be dismissed as complications of the underlying disease in a patient group with significant comorbidity.

\section{Limitations}

We were not able to obtain the incidence of AF for all of the trials which we identified, and the funnel plot shows a relative lack of mid-sized trials, which reflects this. The use of the OOSS from the EMeA scientific discussion may have resulted in an underestimation of the heterogeneity of the data available. Five studies, possibly quite different in various ways from each other, were combined to form a single data set that was treated in the analysis as a single trial. This is likely to result in an overestimation of within-study variance and an underestimation of between-study variance. We have attempted to ameliorate this by performing sensitivity analyses in which the OOSS is excluded.

As the AF data for the largest trials which studied patients with angina, rather than heart failure, were not available separately for each trial, we were unable to definitively assess the effect of treatment indication on $\mathrm{AF}$ risk. It is possible that $\mathrm{AF}$ is less common as a side effect of ivabradine treatment in patients with angina pectoris rather than heart failure. However, a definitive assessment of this risk would require analysis of the individual trial data from the angina studies and possibly even a prospective study of angina patients similar in size to the SHIFT and BEAUTIFUL studies. Such a trial is unlikely to be conducted.

We are unable to provide data comparing the AF risk profile of patients who developed AF to those who did not in either the ivabradine treatment or control groups. It is possible that the increase in AF risk is restricted to a small high-risk subgroup within the overall study population for each trial.

\section{CONCLUSIONS}

Ivabradine treatment is associated with a $15 \%$ increase in the RR of AF. We estimate that 208 patient-years of treatment with ivabradine would be required to cause one new case of AF.

\section{Key messages}

What is known on this subject?

Ivabradine is an effective heart rate lowering treatment which reduces symptoms in chronic stable angina and reduces hospitalisations in chronic heart failure.

Ivabradine may carry an increased risk of atrial fibrillation (AF).

\section{What might this study add?}

Ivabradine treatment carries a substantially higher risk of AF than previously thought; we estimate that 208 patient-years of treatment would be required to cause one new case of $A F$.

\section{How might this impact on clinical practice?}

The risk of AF needs to be taken into consideration when weighing the balance of risk and benefits of ivabradine treatment.

\footnotetext{
Acknowledgements We would like to thank Fail Ageev, Jeff Borer, Ricardo Cappato, Gaetano Lanza, Neeraj Parakh, Anton Potapenko and Giuseppe Rosano for providing additional data.
}

Contributors RIRM and BDK participated in the study design. RIRM performed the search and RIRM and OP extracted the data and translated manuscripts into English. RIRM and MDT performed the analysis. RIRM, BDK and MDT drafted the manuscript. MSK and JPB critically revised the manuscript. All authors participated in the revision and final approval of the manuscript. BDK acts as the guarantor of the manuscript.
Funding RIRM is supported by a British Heart Foundation Clinical Research Training Fellowship. BDK is funded by a British Heart Foundation personal chair. The funder had no role in the design or execution of this study.

\section{Competing interests None.}

Provenance and peer review Not commissioned; externally peer reviewed.

Open Access This is an Open Access article distributed in accordance with the Creative Commons Attribution Non Commercial (CC BY-NC 3.0) license, which permits others to distribute, remix, adapt, build upon this work non-commercially, and license their derivative works on different terms, provided the original work is properly cited and the use is non-commercial. See: http://creativecommons.org/ licenses/by-nc/3.0/

\section{REFERENCES}

1 Difrancesco D. Funny channels in the control of cardiac rhythm and mode of action of selective blockers. Pharmacol Res 2006;53:399-406.

2 Fox K, Ford I, Steg PG, et al. Ivabradine for patients with stable coronary artery disease and left-ventricular systolic dysfunction (BEAUTIFUL): a randomised, double-blind, placebo-controlled trial. Lancet 2008;372:807-16.

3 Swedberg K, Komajda M, Bohm M, et al. Ivabradine and outcomes in chronic heart failure (SHIFT): a randomised placebo-controlled study. Lancet 2010;376:875-85.

4 Ellinor PT, Lunetta KL, Albert CM, et al. Meta-analysis identifies six new susceptibility loci for atrial fibrillation. Nat Genet 2012;44:670-5.

5 den Hoed M, Eijgelsheim M, Esko T, et al. Identification of heart rate-associated loc and their effects on cardiac conduction and rhythm disorders. Nat Genet 2013;45:621-31

6 Haissaguerre $M$, Jais $P$, Shah DC, et al. Spontaneous initiation of atrial fibrillation by ectopic beats originating in the pulmonary veins. $N$ Eng/ J Med 1998;339:659-66.

7 Suenari $K$, Cheng CC, Chen YC, et al. Effects of ivabradine on the pulmonary vein electrical activity and modulation of pacemaker currents and calcium homeostasis. J Cardiovasc Electrophysiol 2012;23:200-6.

8 Camm AJ, Kirchhof P, Lip GY, et al. Guidelines for the management of atrial fibrillation: the Task Force for the Management of Atrial Fibrillation of the European Society of Cardiology (ESC). Europace 2010;12:1360-420.

9 Gregory AP, Dendrou CA, Attfield KE, et al. TNF receptor 1 genetic risk mirrors outcome of anti-TNF therapy in multiple sclerosis. Nature 2012;488:508-11.

10 Moher D, Liberati A, Tetzlaff J, et al. Preferred reporting items for systematic reviews and meta-analyses: the PRISMA statement. BMJ 2009;339:b2535.

11 Higgins JPT, Green S; Cochrane Collaboration. Cochrane handbook for systematic reviews of interventions. Chichester, England; Hoboken, NJ: Wiley-Blackwell, 2008.

12 The R Core Team. R: A Language and Environment for Statistical Computing.

13 Viechtbauer W. Conducting Meta-Analyses in $\mathrm{R}$ with the metafor Package. J Stat Softw 2010;36:1-48.

14 Tardif JC, Ford I, Tendera M, et al. Efficacy of ivabradine, a new selective I(f) inhibitor, compared with atenolol in patients with chronic stable angina. Eur Heart $J$ 2005;26:2529-36.

15 Ruzyllo W, Tendera M, Ford I, et al. Antianginal efficacy and safety of ivabradine compared with amlodipine in patients with stable effort angina pectoris: a 3-month randomised, double-blind, multicentre, noninferiority trial. Drugs 2007;67:393-405.

16 Tardif JC, Ponikowski P, Kahan T. Efficacy of the I(f) current inhibitor ivabradine in patients with chronic stable angina receiving beta-blocker therapy: a 4-month, randomized, placebo-controlled trial. Eur Heart J 2009;30:540-8.

17 Fasullo S, Cannizzaro S, Maringhini G, et al. Comparison of ivabradine versus metoprolol in early phases of reperfused anterior myocardial infarction with impaired left ventricular function: preliminary findings. J Card Fail 2009;15:856-63.

18 Nerla R, Di Franco A, Milo M, et al. Differential effects of heart rate reduction by atenolol or ivabradine on peripheral endothelial function in type 2 diabetic patients. Heart 2012;98:1812-16.

19 Dominguez-Rodriguez A, Consuegra-Sanchez L, Blanco-Palacios G, et al. Anti-inflammatory effects of ivabradine in patients with acute coronary syndrome: a pilot study. Int J Cardiol 2012;158:160-2.

20 Chinchilla A, Daimi H, Lozano-Velasco E, et al. PITX2 insufficiency leads to atrial electrical and structural remodeling linked to arrhythmogenesis. Circ Cardiovasc Genet 2011:4:269-79.

21 Cappato R, Castelvecchio S, Ricci C, et al. Clinical efficacy of ivabradine in patients with inappropriate sinus tachycardia: a prospective, randomized, placebo-controlled, double-blind, crossover evaluation. J Am Coll Cardiol 2012;60:1323-9.

22 Villano A, Di Franco A, Nerla R, et al. Effects of ivabradine and ranolazine in patients with microvascular angina pectoris. Am J Cardiol 2013;112:8-13.

23 Lip GY, Nieuwlaat R, Pisters R, et al. Refining clinical risk stratification for predicting stroke and thromboembolism in atrial fibrillation using a novel risk factor-based approach: the euro heart survey on atrial fibrillation. Chest 2010;137:263-72.

24 Fox K, Komajda M, Ford I, et al. Effect of ivabradine in patients with left-ventricular systolic dysfunction: a pooled analysis of individual patient data from the BEAUTIFUL and SHIFT trials. Eur Heart J 2013;34:2263-70. 